\title{
EDITORIAL
}

\section{Standards of Peer Reviewing}

At the meeting of the Editorial Board last February we were discussing the subject of reviewing of papers, and the question of whether more rigorous standards were being applied to papers describing studies on large (farm) animals was raised following correspondence with an author. This raised another question that is of great concern to all Editors and possibly of even greater concern to authors of papers: how to ensure that the standards of peer reviewing are fair and consistent. One can only deal with consistency within one Journal because it is extremely difficult to consider consistency of the review process across a subject area and without detailed knowledge of the standards set by the other journals publishing nutritional papers. Even within a subject area there are clearly differences between journals which are not necessarily due to differences in scientific standards, but may be because the reviewers or the Editor think a particular area of study or a piece of work is interesting in breaking new ground. One of my former colleagues, the late G. C. Kennedy, once suggested in a light-hearted way that 'if a piece of research was worth doing it was worth doing badly' because at least one would stake a claim for priority in the field and one might find that one had established say $90 \%$ of the truth about a topic.

How does one establish that a common standard is being set for all papers? This, I believe, is a very difficult matter; in fact, I incline to the view that not only is it impossible, in some cases it may not be scientifically desirable. All that I can say is that as far as I can tell by reading all the Editorial Reports and trying to put myself in the position of the authors I do not see any significant bias against papers from any species.

One issue relating to the design of studies does strike me. In many cases those working with large animals may use relatively small numbers of animals in their work, for logistical and resource reasons, so that the statistical design of the study becomes extremely critical. This is the reason why the Institutes which have conducted studies in this area have been largely responsible for setting the standards for statistical design and analysis of nutritional studies. Many human studies on the other hand have an observational character and the design of such studies often cannot be made with the rigour which we expect of those conducting formal experimental studies.

One of the Statistical Editors with whom I used to work during my first term on the Editorial Board once said that he felt that few human studies ever approached the statistical rigour that could be achieved in experimental animal work. To some extent, therefore, one could say that we may appear to be tougher with the large animal researchers.

Some of my colleagues concerned with human nutritional studies will say, and indeed have said, that the British Journal of Nutrition has been unreasonably strict with them. However, the recent correspondence in the British Medical Journal on the standards of clinical research shows that the need to improve statistical standards in human clinical studies is now accepted.

We will continue to maintain our position of giving considerable attention to the statistical design and interpretation in the papers submitted to us. Bear in mind that our peer reviews are conducted by research workers in the relevant fields and their judgements are made against the currently accepted standards in a particular area of nutrition, with the additional input of a Statistical Editor to ensure that the design and analyses are sound. 
While I am on the topic of peer review, some months ago I wrote about the Ethics of Peer Review and, in response to this, Professor Waterlow suggested that open reviewing was one way whereby some of the issues I raised could be avoided. I wonder what you, as authors, reviewers and researchers, feel about this suggestion.

D. A. T. Southgate 